\title{
Comparison of Immunohistochemical Staining for Large T Antigen and Capsid Protein VP1 in BK Polyomavirus-Associated Nephropathy
}

\author{
Kosuke Masutani ${ }^{a}$ b Yuta Matsukuma $^{a} \quad$ Akihiro Tsuchimoto $^{a}$ Yasuhiro Okabe ${ }^{c}$ \\ Atsushi Doi $^{\mathrm{c}}$ Keizo Kaku ${ }^{\mathrm{C}}$ Masafumi Nakamurac ${ }^{\mathrm{C}}$ Toshiaki Nakano ${ }^{\mathrm{a}}$ \\ Kazuhiko Tsuruyad Takanari Kitazono ${ }^{a}$ \\ aDepartment of Medicine and Clinical Science, Graduate School of Medical Sciences, Kyushu University, Fukuoka, \\ Japan; ${ }^{b}$ Division of Nephrology and Rheumatology, Department of Internal Medicine, Fukuoka University, Fukuoka, \\ Japan; 'Department of Surgery and Oncology, Graduate School of Medical Sciences, Kyushu University, Fukuoka,

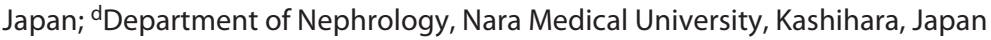

\section{Keywords}

Capsid protein · Cell cycle · Inclusion · Necrosis · Viral replication

\begin{abstract}
Aim: Most transplant centres use SV40 large T antigen (TAg) staining for the diagnosis and assessment of BK polyomavirus-associated nephropathy (BKPyVAN). This study was performed to evaluate the significance of capsid protein VP1 expression in BKPyVAN. Methods: We performed immunohistochemical staining using anti-SV40 TAg and anti-BKPyV VP1 antibodies in 16 index biopsies and 12 re-biopsies of BKPyVAN and compared the patterns of positivity and the percentage of positive tubules by counting whole specimens. We investigated the correlation between serum creatinine increase from baseline and the percentage of positive tubules for both markers in 16 index biopsies. Results: In VP1 staining, positive findings were observed not only in the nuclei of tubular epithelial cells but also in the cytoplasm, cells shedding into the lumen, intra-tubular casts, and in the interstitium. Two of 28 biopsies (7.1\%) showed TAg-positive and VP1-negative results, in which TAg-positive cells were
\end{abstract}

detected only in a single tubule. The median (interquartile range) percentage of positive tubules was 2.8\% (0.7-9.8\%) for TAg and 1.4\% (0.5-3.9\%) for VP1 staining ( $p=0.2)$. In 16 index biopsies, serum creatinine increases significantly correlated with the percentage of VP1-positive tubules ( $r=0.49$, $p=0.02$ ), while this correlation revealed borderline significance with TAg-positive tubules. Conclusions: VP1 expression showed various patterns, but was detected in half as many tubules as TAg staining, which might lead to false negatives in the samples with minimal viral replication. However, increased VP1-positive tubules indicate advanced tubular damage and possible association with graft dysfunction.

(c) 2020 S. Karger AG, Basel

\section{Introduction}

There have been several attempts to create a histological staging system of $\mathrm{BK}$ polyomavirus-associated nephropathy (BKPyVAN) for the prediction of graft failure. A composite system based on viral cytopathic effect, extent of inflammation, and severity of interstitial fibrosis was first proposed by Drachenberg et al. [1], and subse-

karger@karger.com
www.karger.com/nef
Karger


quently the American Society of Transplantation (AST) Infectious Disease Community of Practice published its modifications [2, 3]. However, the AST schema has critical problems; most biopsies are classified into pattern $\mathrm{B}$, and pattern $\mathrm{A}$ is rarely diagnosed, possibly by protocol biopsy. Furthermore, in pattern B, sub-classification into B1, B2, and B3 does not provide sufficient data to discriminate clinical presentations [4]. The Banff Working Group on Polyomavirus Nephropathy performed an indepth analysis with a large retrospective cohort to identify the factors associated with clinical presentation and found 2 significant histological variables: intra-renal viral load (pvl score) and the extent of interstitial fibrosis (ci score). They proposed a new classification in 2013 [5] and recently published the results of a multicentre study [6].

For the evaluation of the pvl score, the Banff Working Group recommends immunohistochemical (IHC) staining for SV40 large T antigen (TAg). However, they have not shown clear data comparing the staining of TAg with that of capsid protein VP1. Previously, Seemayer et al. investigated the expression of TAg and VP1 in BKPyVAN and demonstrated that infected tubular cells in the early and late phases could be distinguished according to the expression of TAg or VP1, p53, Ki67, and nuclear size [7]. That study contributed to the better understanding of the life cycle of BKPyV-infected cells, but the study was based on immunofluorescence staining, and the detailed location of VP1 expression and the ratio of TAg/VP1-positive tubules in the whole biopsy specimen were unclear. Currently, there are commercially available anti-VP1 antibodies which show excellent IHC staining for BKPyV [8]. Thus, we performed an IHC study directly comparing in situ expression of TAg and VP1 in biopsy-proven BKPyVAN.

\section{Materials and Methods}

Study Population and Screening Strategy for BKPyV Infection

Between January 2008 and December 2015, 604 patients received living-donor or deceased-donor kidney transplantation (KT) at Kyushu University Hospital. After KT, screening for BK$\mathrm{PyV}$ infection was performed by urinary cytology testing every 1-3 months until at least 2 years post-transplant. If we found sustained positive urinary decoy cells, we performed polymerase chain reaction (PCR) for BKPyV DNA in plasma, followed by allograft biopsy if we found viraemia or an unexplained rise in serum creatinine ( $\mathrm{sCr}$ ). During the study period, protocol biopsies at 3 and 12 months were also performed. Amongst $604 \mathrm{KT}$ patients, 16 patients $(2.6 \%)$ developed biopsy-proven BKPyVAN. We reviewed baseline $\mathrm{sCr}$, defined as the minimal sCr value within 6 months prior to the index biopsy, and $\mathrm{sCr}$ at diagnosis of $\mathrm{BK}$ PyVAN.

Immunohistochemical Staining for BKPyV

\section{Ethical Approval}

This study was performed in accordance with the guidelines contained within the Declaration of Helsinki. The study protocol was approved by the Human Ethics Committee of Kyushu University Hospital (protocol \#24-54). The ethics committee granted approval to waive the requirement for written informed consent because of the retrospective nature of the study. Consent was also not required because the database was accessed for analytical purposes only and the researchers did not access personal information.

\section{Kidney Allograft Biopsy and Pathological Interpretations}

Biopsy specimens were obtained as 2 or 3 cores using an 18 -gauge biopsy needle under ultrasound guidance. The specimens were fixed with $10 \%$ phosphate buffered formalin and embedded in paraffin wax. Sections were cut at $2 \mu \mathrm{m}$ and stained with haematoxylin-eosin, periodic acid-Schiff, periodic acid-methenamine silver, and elastica-Masson trichrome stains. For the immunofluorescence study, sections were stained with monoclonal antibodies against immunoglobulin (Ig) G, IgA, IgM, complement (C) 3, C1q, C4d, and fibrinogen. The diagnosis of acute and chronic rejection and individual allograft lesions were estimated according to the Banff 2009 classification [9]. IHC staining for SV40 TAg was added for all protocol biopsies and for cause biopsy when the patient showed positive urinary decoy cells, regardless of the results of plasma PCR. The diagnosis of BKPyVAN was made by detecting viral cytopathic effect by light microscopy, positive staining for SV40 TAg, and positive BKPyV DNA in plasma. Histopathological patterns for BKPyVAN were evaluated using the AST classification 2013 [3], accompanied by in situ viral load scoring $[9,10]$.

\section{SV40 TAg and BKPyV VP1 Staining}

Deparaffinised sections were pre-treated for antigen retrieval in a $1,500-\mathrm{W}$ microwave oven for SV40 TAg and by incubation with proteinase $\mathrm{K}$ solution (Roche, Mannheim, Germany) for $\mathrm{BKPyV}$ VP1. The sections were pre-incubated with $5 \%$ non-fat milk for 60 min to eliminate non-specific binding. Then, the sections were incubated with the first antibody (anti-SV40 TAg antibody [1:400, DP02-200UG, mouse monoclonal, Calbiochem, San Diego, CA, USA] and anti-BKV VP1 antibody [1:500, MAB3204M22, mouse monoclonal, Abnova, Taipei, Taiwan]) which were diluted in phosphate buffered saline (PBS)/1\% bovine serum albumin (BSA) overnight at $4^{\circ} \mathrm{C}$. After extensive washing, the sections were incubated with peroxidase-labelled secondary antibody (100 mg/mL; Nichirei Corp., Tokyo, Japan), stained with 3,3' -diaminobenzidine tetrahydrochloride (DAB; Nichirei). Counterstaining was performed with haematoxylin, and sections were coated with glycerol and covered. For negative controls, sections were treated with normal murine IgG (Santa Cruz Biotechnology, Dallas, TX, USA) as the primary antibody.

\section{Histological Evaluation}

Using serial sections, we prepared TAg- and VP1-stained slides as described above from 28 biopsies of 16 BKPyVAN cases. We carefully observed whole specimens, both cortex and medulla, and $\mathrm{BKPyV}$ replication was visually confirmed if the staining reaction was identified in one or more tubular epithelial cells (TECs) per tubular cross section, which was defined as "one positive tubule." We counted all tubular and ductal cross sections at $\times 200$ scanning magnification and calculated the percentage of staining-positive 
tubules. We also evaluated pvl scores: pvl1, $<1 \%$ positive tubules/ ducts; pvl2, 1-10\% positive tubules/ducts; pvl3, $>10 \%$ positive tubules/ducts determined by both SV40 TAg and BKPyV VP1 staining. Importantly, pvl scoring in this study was a little different from that in the multicentre study by the Banff Working Group. They defined a positive tubule as one with intra-nuclear viral inclusion (type 1 or 2 as the most typical variants) by light microscopy and/ or a diagnostic IHC staining reaction for SV40 TAg because tubules with intra-nuclear viral inclusion bodies can, on occasion, be SV40 TAg-negative.

In addition, we classified $26 \mathrm{VP} 1$-positive biopsies into 3 groups according to their VP1 staining patterns: group 1, showing nuclear staining in TECs only $(n=6)$; group 2 , showing cytoplasmic staining accompanied by nuclear staining in TECs $(n=5)$; group 3 , showing positive staining in the intra-tubular casts containing necrotic TECs as well as nuclear and cytoplasmic staining $(n=15)$. We then compared the percentages of VP1-positive tubules amongst these 3 groups.

\section{Statistical Analysis}

Data are expressed as the mean $\pm \mathrm{SD}$, median (interquartile range $[\mathrm{IQR}]$ ), and number positive (\%) as appropriate. JMP version 13.0.0 software (SAS Institute, Cary, NC, USA) was used for all statistical analyses. The Wilcoxon signed-rank test was used to compare the percentage of TAg- and VP1-staining positive tubules. The Wilcoxon signed-rank test followed by the Steel-Dwass test was used to compare the percentages of VP1-positive tubules between groups 1, 2, and 3. Simple regression analysis was used to evaluate the correlation between the percentage of staining-positive tubules and $\mathrm{sCr}$ increase from baseline. A two-tailed $p$ value of $<0.05$ was considered statistically significant.

\section{Results}

Clinical findings at the time of index biopsies in 16 studied cases are summarized in Table 1 . The patients consisted of 12 males and 4 females, with a mean $( \pm$ SD) age of $44 \pm 13$ years old at the time of KT. Kidney allografts were obtained from living donors in 13 cases and from deceased donors in 3 cases. Pre-emptive KT was performed in 3 cases. All patients received basiliximab for induction therapy followed by maintenance immunosuppression: tacrolimus (Tac)/mycophenolate mofetil (MMF)/methylprednisolone (mPSL) in 13 cases, Tac/ everolimus (EVR)/mPSL in 1, cyclosporin $\mathrm{A}(\mathrm{CsA}) /$

Fig. 1. SV40 TAg (a, c) and BKV VP1 (b, d, e, f) positive staining in biopsy-proven BKPyVAN. a Immunohistochemical staining for SV40 TAg revealed clearly positive findings in the nuclei of TECs. b Staining for BKPyV VP1 was also clearly detected in infected cells, but the distribution of positive staining was different from TAg staining. c Detailed observations revealed that TAg was strongly expressed in nuclei of smaller size (arrow), but its expression was weaker in cells with larger nuclei. d VP1 expression was
$\mathrm{MMF} / \mathrm{mPSL}$ in 1 , and $\mathrm{CsA} / \mathrm{MMF} / \mathrm{mPSL}$ in 1 case. $\mathrm{ABO}-$ incompatible $\mathrm{KT}$ was performed in 3 cases, and rituximab administration and plasma exchange were added in those patients. BKPyVAN was diagnosed at a median (interquartile range) of 143 (96-309) postoperative days. Baseline $\mathrm{sCr}$ was $1.4 \pm 0.8 \mathrm{mg} / \mathrm{dL}$, and $\mathrm{sCr}$ at diagnosis was 2.2 $\pm 0.9 \mathrm{mg} / \mathrm{dL}$.

IHC staining for both TAg (Fig. 1a, c) and VP1 (Fig. 1b, $\mathrm{d}-\mathrm{f}$ ) showed excellent staining, but the distribution of positive cells looked different (Fig. 1a, b). TAg-positive staining was clearly observed in the nuclei of TECs, and the staining was strong, especially in the small nuclei. Conversely, staining was weaker in TECs with enlarged nuclei, and this is rarely found in cells shedding into the tubular lumen (Fig. 1c). Another feature was that VP1

Table 1. Clinical characteristics of 16 studied patients with BKPyVAN

Gender (male), $n$ (\%)

$12(75.0)$

Age at KT, years

$44 \pm 13$

Donor source (living donor), $n$ (\%) 13 (81.2)

Pre-emptive KT, $n(\%)$

$3(18.8)$

ABO-incompatible KT, $n$ (\%)

HLA-mismatch count (A, B, and DR)

$3(18.8)$

Primary kidney disease, $n(\%)$

Glomerulonephritis

$2.9 \pm 1.0$

Diabetes

$11(68.8)$

Others

$2(12.4)$

$3(18.8)$

Basiliximab use, $n(\%)$

$16(100)$

Rituximab use, $n(\%)$

3 (18.8)

Maintenance immunosuppression, $n(\%)$

$\mathrm{Tac} / \mathrm{MMF} / \mathrm{mPSL}$

$13(81.2)$

Tac/EVR/mPSL

$1(6.3)$

CsA/MMF/mPSL

$1(6.3)$

CsA/EVR/mPSL

$1(6.3)$

Postoperative days, median (interquartile range) 143 (96-309)

Baseline sCr, mg/dL

$1.4 \pm 0.8$

$\mathrm{sCr}$ at diagnosis, $\mathrm{mg} / \mathrm{dL}$

$2.2 \pm 0.9$

BKPyVAN, BK polyomavirus-associated nephropathy; KT, kidney transplantation; HLA, human leucocyte antigen; Tac, tacrolimus; MMF, mycophenolate mofetil; mPSL, methylprednisolone; EVR, everolimus; CsA, cyclosporin A; sCr, serum creatinine.

negative in TECs with strong TAg expression, but positive in both nuclei and cytoplasm in TECs with larger nuclei or severely damaged TECs (arrow). e VP1-positive material showed leakage from the point of disruption of the tubular basement membrane (arrow) and was found in the interstitium. $f$ Intra-tubular casts consisting of necrotic debris also showed positive staining for VP1. TAg, large T antigen; BKPyVAN, BK polyomavirus-associated nephropathy; TECs, tubular epithelial cells.

(For figure see next page.)
Masutani et al. 

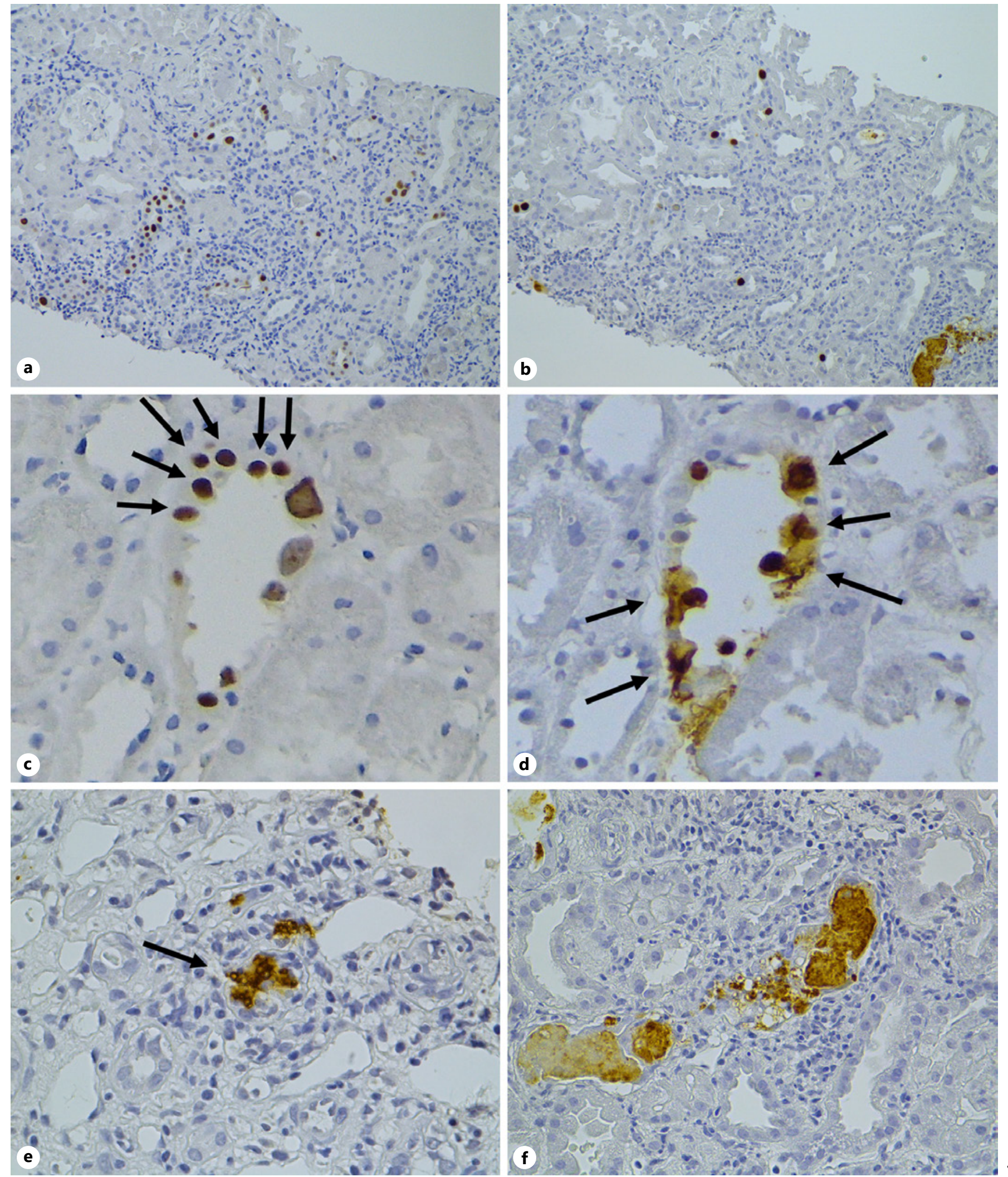

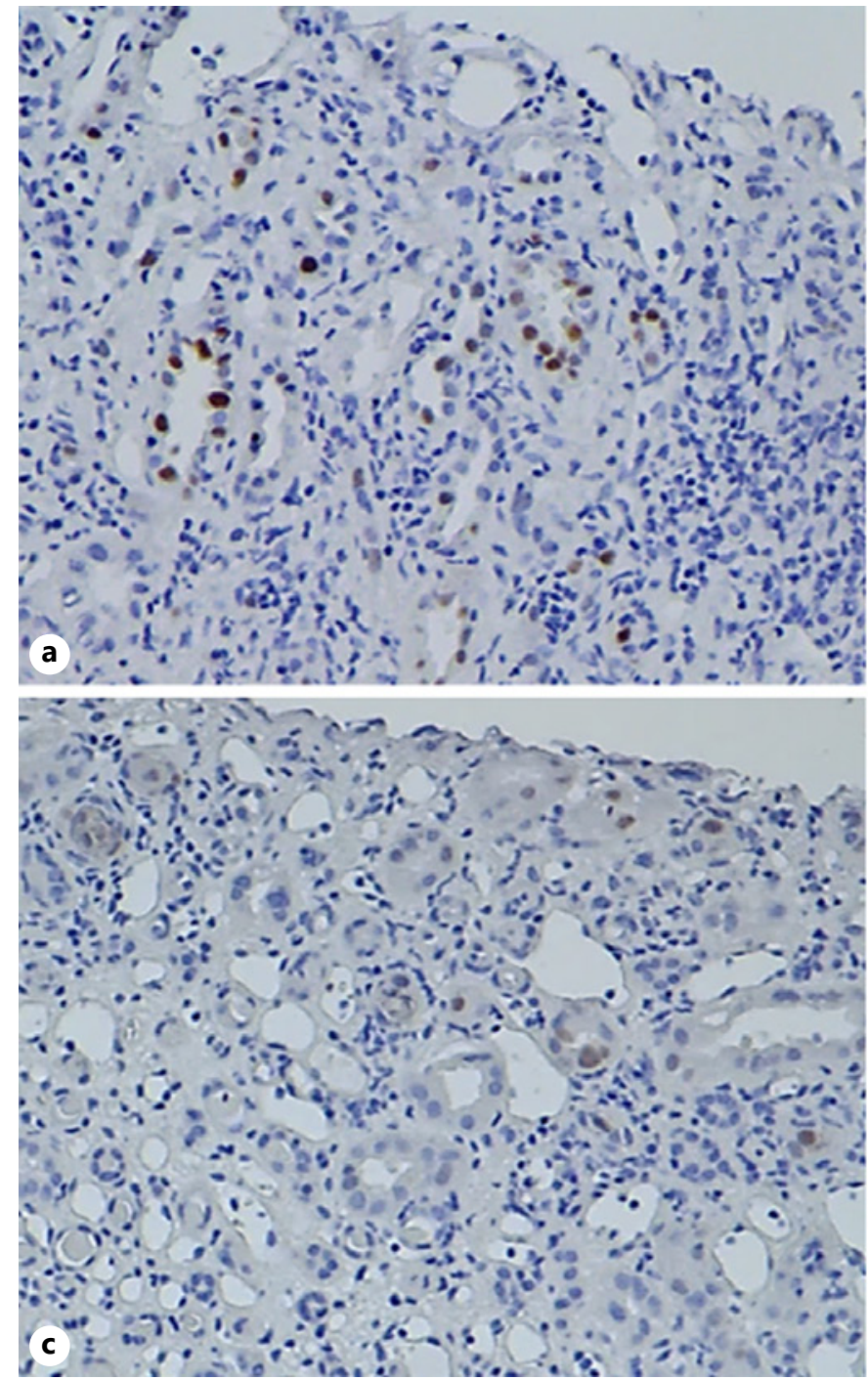

Fig. 2. Representative findings of TAg predominant area indicating early phase of infection $(\mathbf{a}, \mathbf{b})$ and VP1 predominant area indicating late phase of infection $(\mathbf{c}, \mathbf{d})$ in the same biopsy sample. An area showing predominant TAg staining (a) without VP1 staining (b) was observed, suggesting early phase of infection. In a second area of this biopsy sample, we could identify predominant VP1

staining showed various findings; nuclei and cytoplasm (Fig. 1d), interstitium near the infected tubule with disruption of the tubular basement membrane (Fig. 1e), and intra-tubular casts (Fig. 1f) were positive. As demonstrated in the previous study by Seemayer et al. [7], it seemed to be possible to discriminate the $\mathrm{BKPyV}$-infected tubules/areas in the early phase (Fig. 2a, b) and late phase (Fig. 2c, d) by the balance of TAg and VP1 staining intensity.
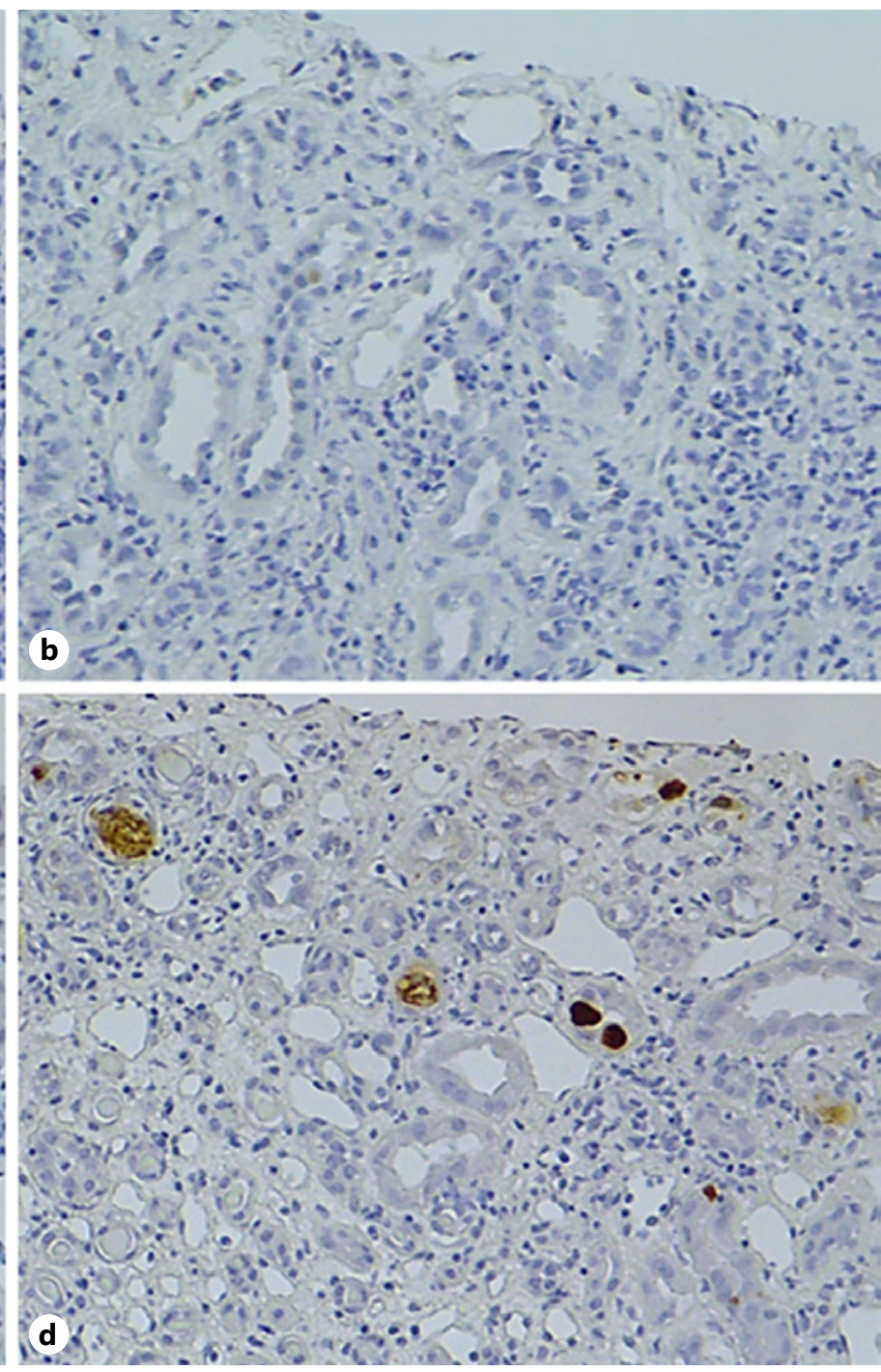

staining (d) compared with weak TAg staining (c). In the latter area, VP1-positive intra-tubular casts were also observed (d), suggesting late phase of infection with tubular cell necrosis and shedding into the lumen. SV40 TAg staining (a, c); BKPyV VP1 staining (b, d). TAg, large T antigen; BKPyVAN, BK polyomavirusassociated nephropathy.

With regard to the visually counted IHC stainingpositive tubules, we counted $1,276 \pm 737$ tubules in TAg-stained sections and 1,209 \pm 646 in the VP1stained sections in 28 biopsies. The percentage of staining-positive tubules was $2.8 \%(0.7-9.8 \%)$ for TAg and $1.4 \%(0.5-3.9 \%)$ for VP1 staining. Although those percentages did not reach statistical significance $(p=0.2)$, VP1 staining detected half as many positive tubules as TAg staining (Fig. 3a). The pvl score determined by 


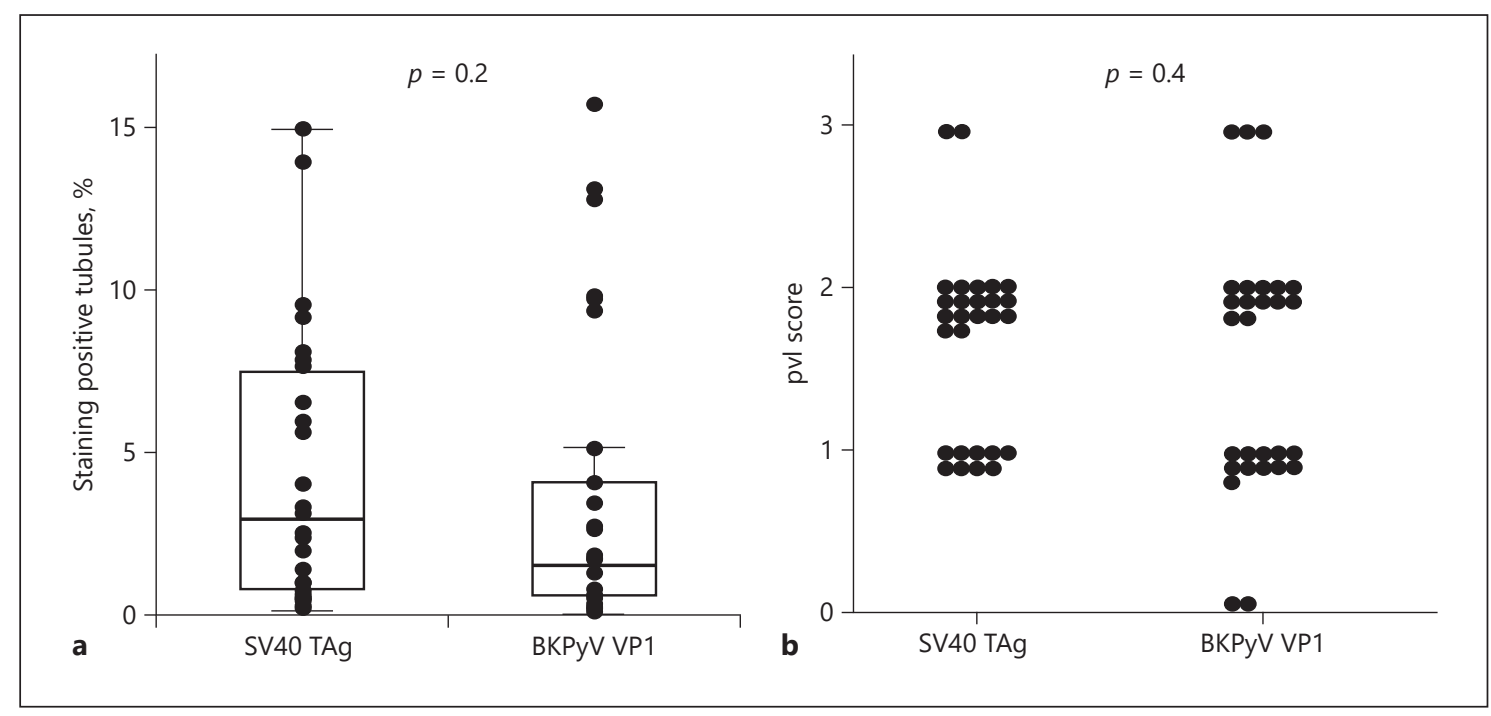

Fig. 3. Percentages of TAg- and VP1-positive tubules (a) and pvl scores (b) determined by staining for both TAg and VP1. a The visually counted percentage of staining-positive tubules was not statistically different between TAg and VP1 staining. $\mathbf{b}$ The distribution of pvl scores determined by dual staining was also not different, but 2 biopsies showed TAg-positive and VP1-negative results. TAg, large T antigen.

IHC staining for TAg and VP1 revealed no statistical difference between TAg and VP1 staining $(p=0.4$, Fig. 3b). There were 2 biopsies showing TAg-positive, VP1-negative results. In those biopsies, the detection of TAg-positive tubules was also very difficult because there was only a single positive tubule found throughout the specimen.

Further observation focussing on the staining pattern and the percentage of positive tubules for VP1 revealed that the biopsy samples in group 3 showed a significantly higher percentage of positive tubules than those in group 1 (median of 3.3 vs. $0.5 \%, p<0.01$ ) and tended to be higher than those in group 2 (3.3 vs. $0.6 \%, p=0.07$ ) (Fig. 4). We also analyzed the correlation between staining-positive tubules (\%) and $\mathrm{sCr}$ increase from baseline using 16 index biopsies. In this analysis, we excluded 12 follow-up biopsies because temporal increase in $\mathrm{sCr}$ and decreased staining-positive tubules would be observed after the reduction of immunosuppression, and this could confound the results [11]. As shown in Figure 4, sCr increase significantly correlated with the percentage of VP1 stainingpositive tubules $(r=0.49, p=0.02$; Fig. $5 \mathrm{a})$, while the correlation revealed borderline significance with TAg-positive tubules (Fig. 5b).

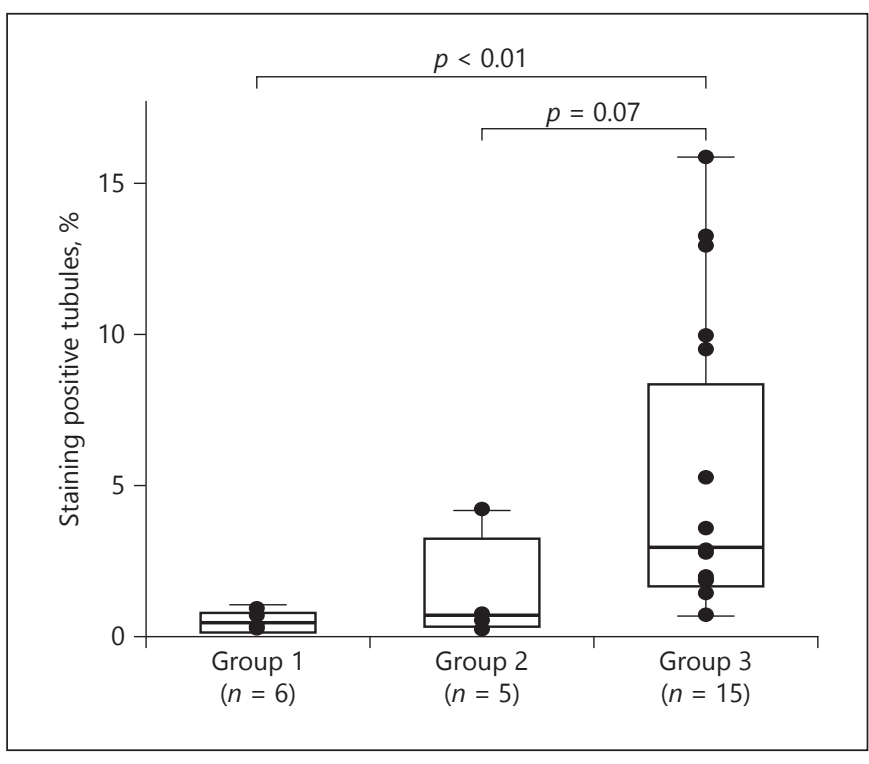

Fig. 4. Percentage of VP1-positive tubules in the 3 groups classified by the pattern of positive staining. The percentage of VP1-positive tubules in group 3 was significantly higher than that in group 1 $(p<0.01)$ and tended to be higher than that in group $2(p=0.07)$. We classified 26 VP1-positive biopsies into 3 groups according to their VP1 staining patterns: group 1, showing nuclear staining in TECs only $(n=6)$; group 2 , showing cytoplasmic staining accompanied by nuclear staining in TECs $(n=5)$; group 3 , showing positive staining in the intra-tubular casts containing necrotic TECs as well as nuclear and cytoplasmic staining $(n=15)$. TAg, large T antigen; TECs, tubular epithelial cells. 


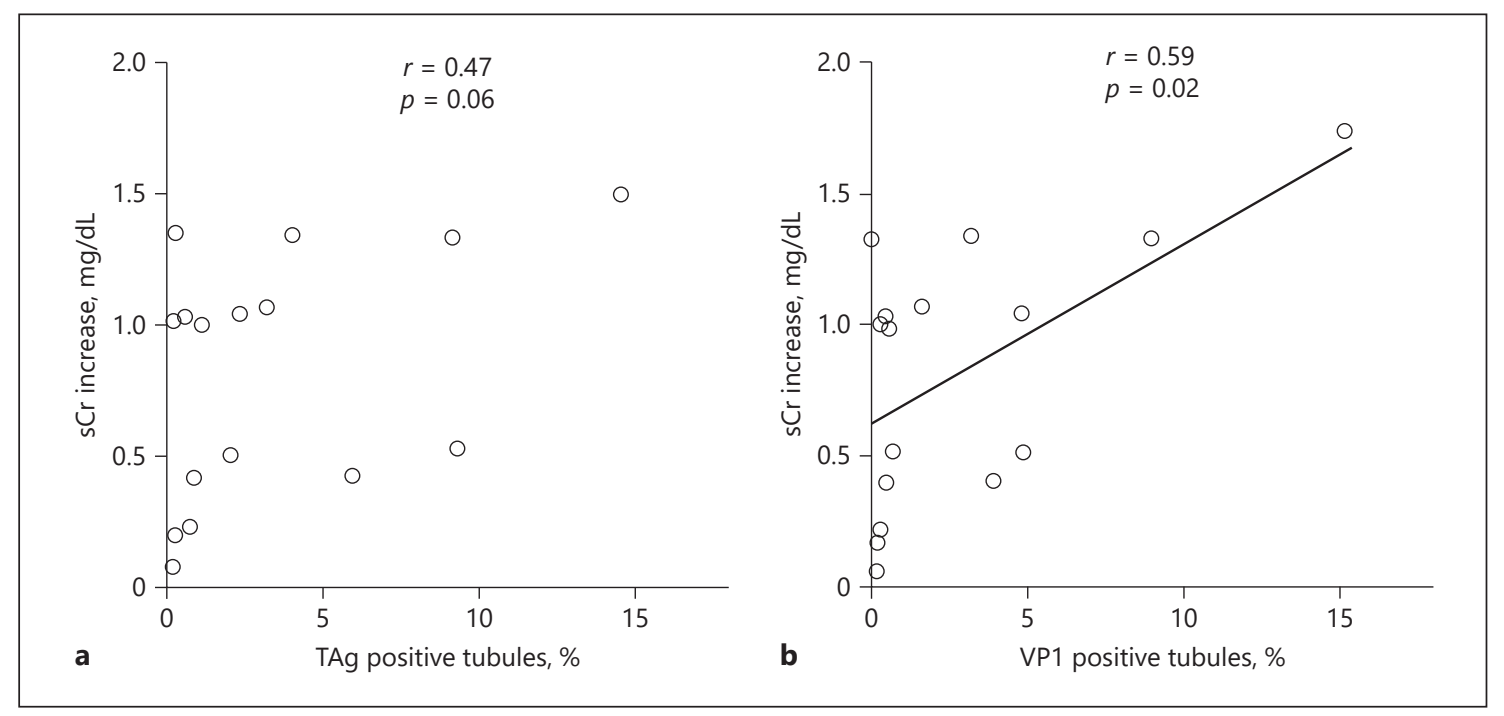

Fig. 5. Correlation between serum creatinine increase from baseline and the percentage of staining-positive tubules in TAg (a) and VP1 staining (b). Increase of serum creatinine significantly correlated with the percentage of VP1-positive tubules $(r=0.59, p=0.02)(\mathbf{b})$, but the correlation with the percentage of TAg-positive tubules revealed borderline significance $(r=0.47, p=0.06)(\mathbf{a})$. TAg, large T antigen; sCr, serum creatinine.

\section{Discussion}

In this study, we performed IHC staining using TAg and VP1 antibodies to demonstrate the detailed expression of VP1. We also compared the expression of these 2 antigens and investigated the clinical presentation in BKPyVAN at diagnosis. IHC staining revealed that TAg and VP1 positivity was located in different cells in a single tubule and appeared in cells with different nuclear size, suggesting early and late phases of infection. This was consistent with previous studies [7]. Different from the immunofluorescence study, however, IHC staining further identified that VP1-positive material, possibly viral aggregates, was also observed in the cytoplasm of TECs and intra-tubular casts containing shed TECs and showed interstitial leakage from the disrupted tubular basement membrane. In addition, biopsies with VP1-positive intratubular casts suggest lysis, necrosis, and shedding into the lumen of TECs, indicating more advanced disease, and this was clearly associated with a higher percentage of VP1-positive tubules than in biopsies with nuclear staining only. These findings have not been demonstrated previously, and our results clearly support the suggested process of progressive $\mathrm{BKPyV}$ infection in the kidney allograft [12].

For the diagnosis of BKPyVAN, the Banff Working Group recommends SV40 TAg staining based on the re- sults of the multicentre study investigating inter-laboratory reproducibility by the Banff Initiative for Quality Assurance in Transplantation [13]. Amongst 78 participating centres, 2 institutes used antibodies directed against VP1, and false negative results were found in the positive control samples in those centres. However, these false negatives were found in glass slides with a SV40-infected monkey fibroblast-like cell line, not in human BKPyVAN or human renal cell carcinoma positive for BKPyV [13]. Thus, we considered the necessity of a clear and direct comparison of TAg and VP1 staining to re-evaluate whether VP1 staining could cause false negative results in BKPyVAN. Despite the lack of statistical significance, the percentage of VP1-positive staining was found in half as many tubules as TAg staining (median of 1.4 vs. $2.8 \%$, respectively). In addition, 2 of 28 biopsies (7.1\%) showed TAg-positive and VP1-negative results. These 2 samples were difficult to diagnose using the suggested methods because they were early disease with only a single tubule showing positive SV40 TAg expression. VP1 staining could not detect such early disease, and TAg staining is superior to VP1 for optimal diagnostic workup.

For predicting graft failure, the Banff Working Group proposed the first staging system in 2009 . The main difference from the AST schema was that it consisted of acute tubular injury (TEC shedding and denudation of the tubular basement membrane) instead of interstitial 
inflammation $[9,10]$. As a result of their multicentre study, stage $\mathrm{C}$ disease (ci score 3 with any degree of TEC injury) showed the worst graft outcome, but the difference between stage A and stage B diseases was not significant. Thus, the schema did not reach any conclusion and was not incorporated in the Banff classification [14]. In the new Banff schema proposed in 2013, histological class is determined by the combination of ci and pvl scores. Retrospective observation revealed that it seems to have a discriminating power for $\mathrm{sCr}$ changes from baseline at 12 and 24 months. However, sCr change in class 1 and class 2 groups was small; the incidence of resolution (defined as the first re-biopsy without nephropathy and/ or clearance of viraemia) was not different. Although significant differences in the graft loss rate amongst the 3 groups were found, the multiple comparisons between class 1 and class 2 was not described. To confirm the usefulness of the schema, further validation is necessary.

It is also known that graft dysfunction at diagnosis is a strong predictor of graft loss $[4,15]$, and in this study, the percentage of VP1-positive tubules in 16 index biopsies significantly correlated with $\mathrm{sCr}$ increase from baseline to diagnosis. Lysis, necrosis, and shedding of TECs are the features of advanced tubular damage $[9,16]$, and in our IHC study, TEC shedding into the lumen was strongly associated with nuclear and cytoplasmic VP1 expression; therefore, the association between VP1 expression and graft dysfunction seems to be reasonable. In this regard, the pvl score determined by VP1 staining and TAg/VP1positive cell ratio as novel variables for prediction of late graft function are of interest.

There are several potential limitations in this study. Most importantly, the small sample size and the lack of analysis of late graft function due to short-term follow-up diminished the results. The pvl scores determined by TAg and VP1 staining were not identical but showed small differences. Thus, we could not find any difference in clinical presentation. Another limitation is that the estimation of $\mathrm{BKPyV}$-infected tubules by visual counting is not practical for everyday practice. The current 3-titre scoring system of pvl scoring also depends on the visual estimation of staining-positive tubules combined with nuclear inclusion occasionally negative for SV40 TAg. The current computer-aided image analysis cannot detect stainingpositive "tubules" but can detect "nuclei" as a stained particle. The development of digital pathology combined with artificial intelligence might solve this problem in the future.

In summary, BKPyV VP1 staining showed clearly positive findings and various patterns, but it detected half as many tubules as SV40 TAg staining, which might lead to false negatives in samples with minimal BKPyV replication. Thus, for diagnosis, TAg staining is superior to VP1 staining. Conversely, increased VP1-positive tubules indicate advanced tubular damage and may have a possible association with graft dysfunction.

\section{Acknowledgements}

The authors thank Ms. H. Hayashida for her excellent technical assistance. They also thank Gillian Campbell, PhD, from Edanz Group (www.edanzediting.com/ac) for editing a draft of this manuscript.

\section{Statement of Ethics}

Participants in this study have given written informed consent, and the study protocol was approved by the Human Ethics Committee of Kyushu University Hospital. The study was conducted in accordance with the Helsinki Declaration.

\section{Conflict of Interest Statement}

The authors have no conflicts of interest to declare.

\section{Funding Sources}

This work was supported by JSPS KAKENHI (Grant No. JP16K11066).

\section{Author Contributions}

K.M., Y.M., and A.T. designed the study. Y.O., A.D., and K.K. performed the kidney transplantations and allograft biopsies. Y.M. and A.T. assessed the histological findings. T.N. and K.T. performed the data collection and analysis. K.M. wrote the manuscript. M.N., K.T., and T.K. critically revised the manuscript for important intellectual content.

\begin{tabular}{|c|c|}
\hline References & $\begin{array}{l}1 \text { Drachenberg RC, Drachenberg CB, Papad- } \\
\text { imitriou JC, Ramos E, Fink JC, Wali R, et al. } \\
\text { Morphological spectrum of polyoma virus } \\
\text { disease in renal allografts: diagnostic accuracy } \\
\text { of urine cytology. Am J Transplant. 2001;1(4): } \\
373-81 \text {. } \\
2 \text { Hirsch HH, Randhawa P; AST Infectious Dis- } \\
\text { ease Community of Practice. BK virus in solid } \\
\text { organ transplant recipients. Am J Transplant. } \\
\text { 2009;9(Suppl 4):S136-46. }\end{array}$ \\
\hline
\end{tabular}

Nephron 2020;144(suppl 1):28-36 DOI: $10.1159 / 000510967$ 
3 Hirsch HH, Randhawa P; AST Infectious Disease Community of Practice. BK polyomavirus in solid organ transplantation. Am J Transplant. 2013;13(Suppl 4):179-88.

4 Masutani K, Shapiro R, Basu A, Tan H, Wijkstrom M, Randhawa P. The Banff 2009 working proposal for polyomavirus nephropathy: a critical evaluation of its utility as a determinant of clinical outcome. Am J Transplant. 2012;12(4):907-18.

5 Haas M, Sis B, Racusen LC, Solez K, Glotz D, Colvin RB, et al. Banff 2013 meeting report: inclusion of C4d-negative antibody-mediated rejection and antibody-associated arterial lesions. Am J Transplant. 2014;14(2):272-83.

6 Nickeleit V, Singh HK, Randhawa P, Drachenberg $\mathrm{CB}$, Bhatnagar R, Bracamonte $\mathrm{E}$, et al. The Banff Working Group Classification of Definitive Polyomavirus Nephropathy: morphologic definitions and clinical correlations. J Am Soc Nephrol. 2018;29(2):680-93.

7 Seemayer CA, Seemayer NH, Dürmüller U, Gudat F, Schaub S, Hirsch HH, et al. BK virus large $\mathrm{T}$ and VP-1 expression in infected human renal allografts. Nephrol Dial Transplant. 2008;23(12):3752-61.
8 Alexiev BA, Papadimitriou JC, Drachenberg CB. BK polyomavirus-infected nephrogenic adenoma of the urinary bladder in a renal transplant recipient: a case report. Pathol Res Pract. 2015;211(9):697-701.

9 Sis B, Mengel M, Haas M, Colvin RB, Halloran PF, Racusen LC, et al. Banff '09 meeting report: antibody mediated graft deterioration and implementation of Banff working groups. Am J Transplant. 2010;10(3):464-71.

10 Sar A, Worawichawong S, Benediktsson $\mathrm{H}$, Zhang J, Yilmaz S, Trpkov K. Interobserver agreement for polyomavirus nephropathy grading in renal allografts using the working proposal from the 10th Banff Conference on Allograft Pathology. Hum Pathol. 2011; 42(12):2018-24.

11 Masutani K, Tsuchimoto A, Matsukuma Y, Kurihara K, Nishiki T, Kitada H, et al. Temporal serum creatinine increase and exacerbation of tubulointerstitial inflammation during the first two months in resolving polyomavirus BK nephropathy. Nephrology. 2015; 20(Suppl 2):45-50.
12 Lamarche C, Orio J, Collette S, Senécal L, Hébert MJ, Renoult É, et al. BK polyomavirus and the transplanted kidney: immunopathology and therapeutic approaches. Transplantation. 2016;100(11):2276-87.

13 Adam B, Randhawa P, Chan S, Zeng G, Regele $\mathrm{H}$, Kushner YB, et al. Banff initiative for quality assurance in transplantation (BIFQUIT): reproducibility of polyomavirus immunohistochemistry in kidney allografts. Am J Transplant. 2014;14(9):2137-47.

14 Nishi S. Polyomavirus nephropathy -recent pathological diagnostic problems and the report from the 2011 Banff meeting. Clin Transplant. 2012;26(Suppl 24):9-12.

15 Nankivell BJ, Renthawa J, Sharma RN, Kable K, O'Connell PJ, Chapman JR. BK virus nephropathy: histological evolution by sequential pathology. Am J Transplant. 2017;17(8): 2065-77.

16 Drachenberg CB, Hirsch HH, Ramos E, Papadimitriou JC. Polyomavirus disease in renal transplantation: review of pathological findings and diagnostic methods. Hum Pathol. 2005;36(12): 1245-55. 